\title{
Dépôt au sol, dans la Grèce du nord, de césium 134 et de césium 137, à la suite de l'accident de Tchernobyl ${ }^{\star}$
}

\author{
M. ANTONOPOULOS-DOMIS, A. CLOUVAS et P. TERVISIDIS**
}

(Manuscrit reçu le 23 décembre 1987)

\begin{abstract}
RÉSUMÉ
Par spectrométrie $\gamma$, nous avons mesuré la concentration de ${ }^{134} \mathrm{Cs}$ et de ${ }^{137} \mathrm{Cs}$, dans le sol de la Grèce du nord, à des profondeurs comprises entre 0 et $10 \mathrm{~cm}$. Nous en avons déduit le dépôt de ces radionucléides à la suite de l'accident de Tchernobyl. Bien que les valeurs soient assez divergentes pour des distances faibles, nous en déduisons une valeur moyenne du dépôt de ${ }^{134} \mathrm{Cs}$ et ${ }^{137} \mathrm{Cs}$ de l'ordre de $20 \mathrm{kBq} \mathrm{m}^{-2}$, sur le sol de la Grèce du nord. Nous avons comparé nos résultats à la radioactivité naturelle de ${ }^{40} \mathrm{~K}$ dans le sol. De la distribution de la concentration de ${ }^{137} \mathrm{Cs}$ en fonction de la profondeur dans le sol, nous concluons que la majeure partie $(95 \%)$ du dépôt de radiocésium est piégée dans les premiers $10 \mathrm{~cm}$ du sol non perturbé.
\end{abstract}

\section{ABSTRACT}

The deposition of ${ }^{137} \mathrm{Cs}$ and ${ }^{134} \mathrm{Cs}$ in Northern Greece after the Chernobyl accident was indirectly determined from properly performed soil measurements. Even if radiocesium deposition was found to be an extremely local parameter, we can attribute a mean value for Northern Greece of the order of $20 \mathrm{kBq} \mathrm{m}^{-2}$. The $95 \%$ of this deposit is found in the first $10 \mathrm{~cm}$ of the undisturbed soil.

\section{INTRODUCTION}

A la suite de l'accident de Tchernobyl, le dépôt de ${ }^{134} \mathrm{Cs}$ et de ${ }^{137} \mathrm{Cs}$ sur le sol de la Grèce était hétérogène, avec des minimums de l'ordre de $1 \mathrm{kBq} \mathrm{m}^{-2}$, et des maximums allant jusqu'à $90 \mathrm{kBq} \mathrm{m}^{-2}$. A cause de cette

* Communication présentée lors du congrès franco-italien SFRP-Associazione italiana di protezione contro le radiazioni, Castelgandolfo (Italie), 12-13 octobre 1987. Grèce.

** Département de génie électrique, Université de Thessaloniki, GR 54006 Thessaloniki, 
inhomogénéité, les quelques valeurs [1] des dépôts de radiocésium, mesurées directement au début de mai 1986, malgré leur importance primordiale, ne sont pas suffisantes pour donner une bonne description du dépôt, particulièrement pour la Grèce du nord où il est plus important. Dans tous les modèles mathématiques [4], les valeurs du dépôt au sol de radiocésium sont très importantes pour prévoir la pollution alimentaire par ces radionucléides à longue durée de vie. Nous avons donc été amenés à faire une étude systématique de ces dépôts sur le sol de la Grèce du nord.

\section{MÉTHODE EXPÉRIMENTALE}

De février à juin 1987, nous avons recueilli 150 échantillons de terre non perturbée, dans 15 départements de la Grèce du nord. Les échantillons ont été collectés sur des terrains sans végétation, et à une profondeur allant jusqu'à $10 \mathrm{~cm}$, à l'aide d'un tube cylindrique de section donnée $\left(6 \mathrm{~cm}^{2}\right)$. Comme nous le verrons au paragraphe suivant, la profondeur de $10 \mathrm{~cm}$ est suffisante pour que nous puissions considérer que la majeure partie du césium déposée est piégée dans cette couche. Durant l'échantillonnage, nous mesurions l'exposition externe au moyen de scintillateurs de Nal portables; le but était d'éviter des endroits "chauds" formés artificiellement (par exemple, accumulation d'eau de pluie, etc); cela aurait donné une fausse image du dépôt.

Nous avons mesuré l'activité de chaque échantillon en ${ }^{137} \mathrm{Cs}$ et ${ }^{134} \mathrm{Cs}$ par spectrométrie $\gamma$ au moyen d'un dispositif expérimental classique. De ces valeurs, nous avons déduit le dépôt de ces radionucléides sur le sol. En effet, avant Tchernobyl, la quantité de radiocésium dans le sol de la Grèce du nord, due aux essais nucléaires, est inférieure d'un ordre de grandeur; on peut la négliger.

\section{RÉSULTATS, DISCUSSION}

Le dépôt de ${ }^{134} \mathrm{Cs}$ et ${ }^{137} \mathrm{Cs}$ après Tchernobyl dépend de la concentration de ces radionucléides dans l'air et des conditions météorologiques lorsque le nuage radioactif a survolé la Grèce, notamment l'existence de pluies. La plus grande partie du dépôt de ${ }^{134} \mathrm{Cs}$ et ${ }^{137} \mathrm{Cs}$ dans la région de Thessalonique est due à la pluie intense du 5 mai 1986; cela se déduit de nos mesures de la radioactivité de l'air et de l'exposition externe. Nous observons, le 5 mai 1986, une diminution importante de la radioactivité de l'air (fig. 1), en même temps qu'une augmentation équivalente de l'exposition externe. Cette figure 1 montre que la radioactivité de l'air dans la région de Thessalonique est redevenue normale après le 10 mai 1986. A quelques jours près, cela est vrai pour toutes les autres régions de la Grèce du nord, où nous avons fait des mesures semblables. La concentration en radiocésium dans la terre de la Grèce du nord est due, en majorité, au dépôt de ces radionucléides sur le sol, durant les quinze jours qui ont suivi l'accident. 


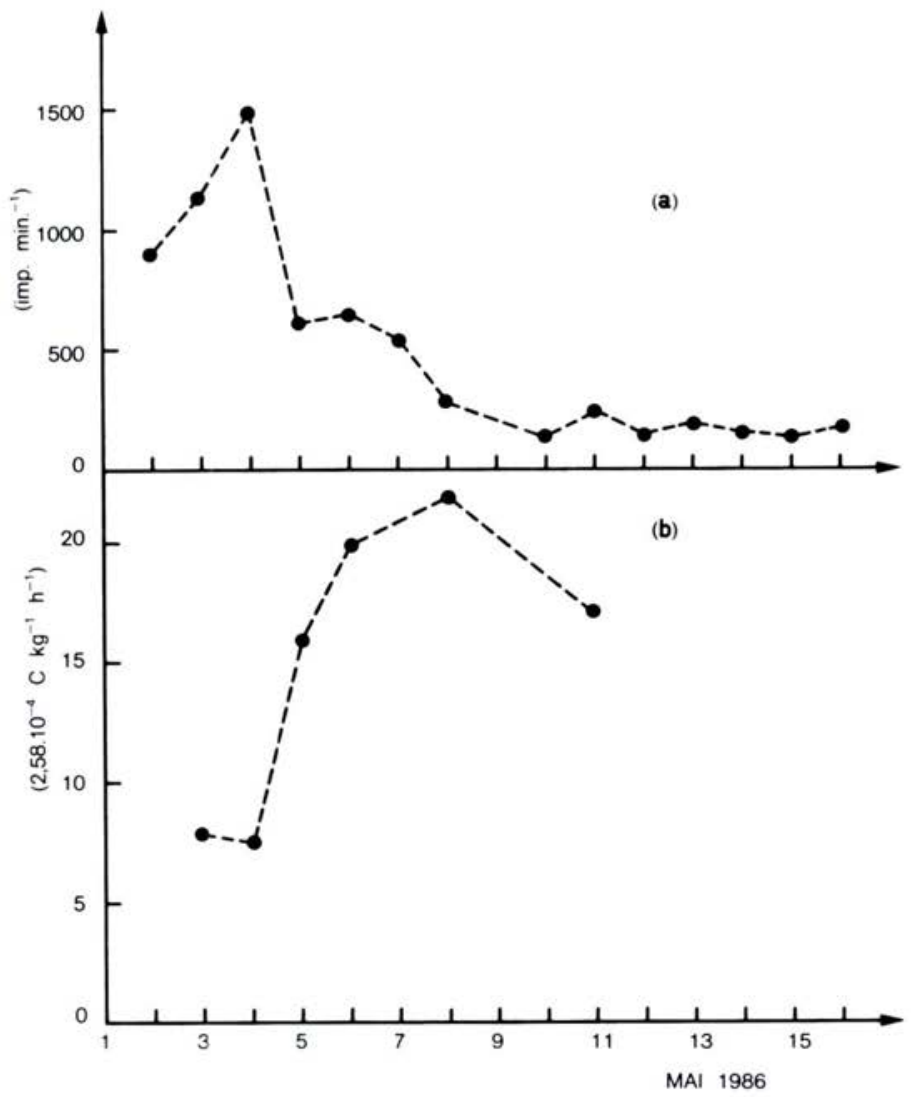

Fig. 1. - (a) Radioactivité de l'air. (b) Débit d'exposition à $1 \mathrm{~m}$ au-dessus du sol. Valeurs journalières moyennes, lieu: école polytechnique de Thessalonique.

La figure 2 présente le dépôt de ${ }^{134} \mathrm{Cs}$ et ${ }^{137} \mathrm{Cs}\left(\mathrm{kBq} \mathrm{m}^{-2}\right)$ sur le sol de 15 départements de la Grèce du nord. Les valeurs sont rapportées au $1^{\text {er }}$ mai 1986; pour chaque département, elles sont la moyenne de 9 mesures indépendantes faites en des lieux différents. L'erreur absolue de nos mesures est de l'ordre de $20 \%$, l'erreur relative est inférieure à $5 \%$. II est intéressant de comparer nos valeurs à celles obtenues par des mesures directes; en effet, le service de radioprotection de l'environnement du centre "Democritos" a mesuré, de manière directe, en mai 1986 , les dépôts de ${ }^{134} \mathrm{Cs}$ et ${ }^{137} \mathrm{Cs}$ autour de l'école polytechnique de Thessalonique. Les valeurs obtenues de $12 \mathrm{kBq} \mathrm{m}^{-2}\left({ }^{137} \mathrm{Cs}\right)$ et $6 \mathrm{kBq} \mathrm{m}^{-2}\left({ }^{134} \mathrm{Cs}\right)$ sont en bon accord avec les nôtres $\left(15 \mathrm{kBq} \mathrm{m}^{-2}\right.$ et $\left.8 \mathrm{kBq} \mathrm{m}^{-2}\right)$. Dans toutes nos mesures, le dépôt de ${ }^{134} \mathrm{Cs}$ est presque la moitié de celui de ${ }^{137} \mathrm{Cs}$. Bien que les valeurs soient 
assez divergentes pour des distances relativement courtes, nous attribuons au dépôt de ${ }^{134} \mathrm{Cs}$ et ${ }^{137} \mathrm{Cs}$ sur le sol de la Grèce du nord une valeur de l'ordre de $20 \mathrm{kBq} \mathrm{m}^{-2}$, trois fois plus élevée que la valeur moyenne pour toute la Grèce [2].

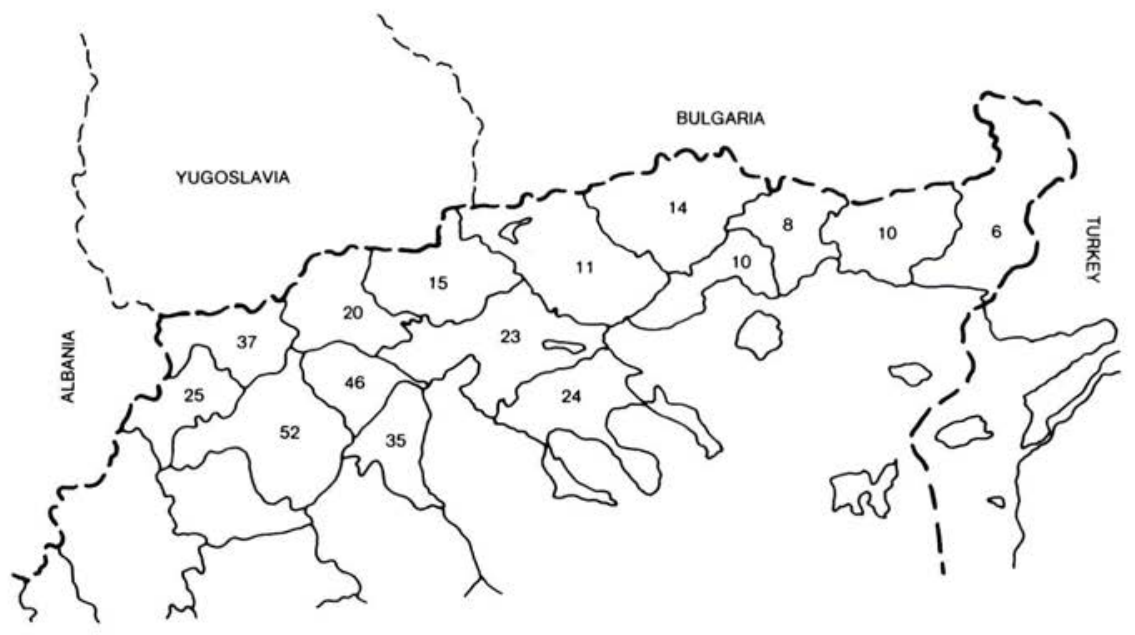

Fig. 2. - Dépôt de ${ }^{137} \mathrm{Cs}$ et ${ }^{134} \mathrm{Cs}\left(\mathrm{kBq} \mathrm{m}^{-2}\right)$ sur le sol de la Grèce du nord, après Tchernobyl.

Ainsi que nous l'avons mentionné plus haut, pour la validité des mesures, il était nécessaire de vérifier que la plus grande partie du radiocésium se trouvait piégée dans les 10 premiers centimètres. Les mesures de distribution de la concentration du radiocésium en fonction de la profondeur montrent [3] que la majorité de celui-ci se trouve dans les premiers centimètres, mais ces distributions dépendent fortement de la nature du sol. II fallait donc mesurer ces distributions pour les sols de la Grèce du nord. La distribution de la concentration du radiocésium en fonction de la profondeur peut être caractérisée, en général, par une décroissance exponentielle de la forme:

$$
A(x)=A_{0} \exp (-x / d)
$$

$\mathrm{A}(\mathrm{x})$ étant l'activité de ${ }^{137} \mathrm{Cs}$ par unité de masse $\left(\mathrm{Bq} \mathrm{kg}^{-1}\right.$ à la profondeur $\left.\mathrm{x}\right)$. La figure 3 représente la fonction mesurée:

$$
B(x)=\int_{x-5}^{x} A(x) d x
$$

Pour les sols de la Grèce du nord étudiés, d varie entre $2 \mathrm{~cm}$ et $5 \mathrm{~cm}$. 


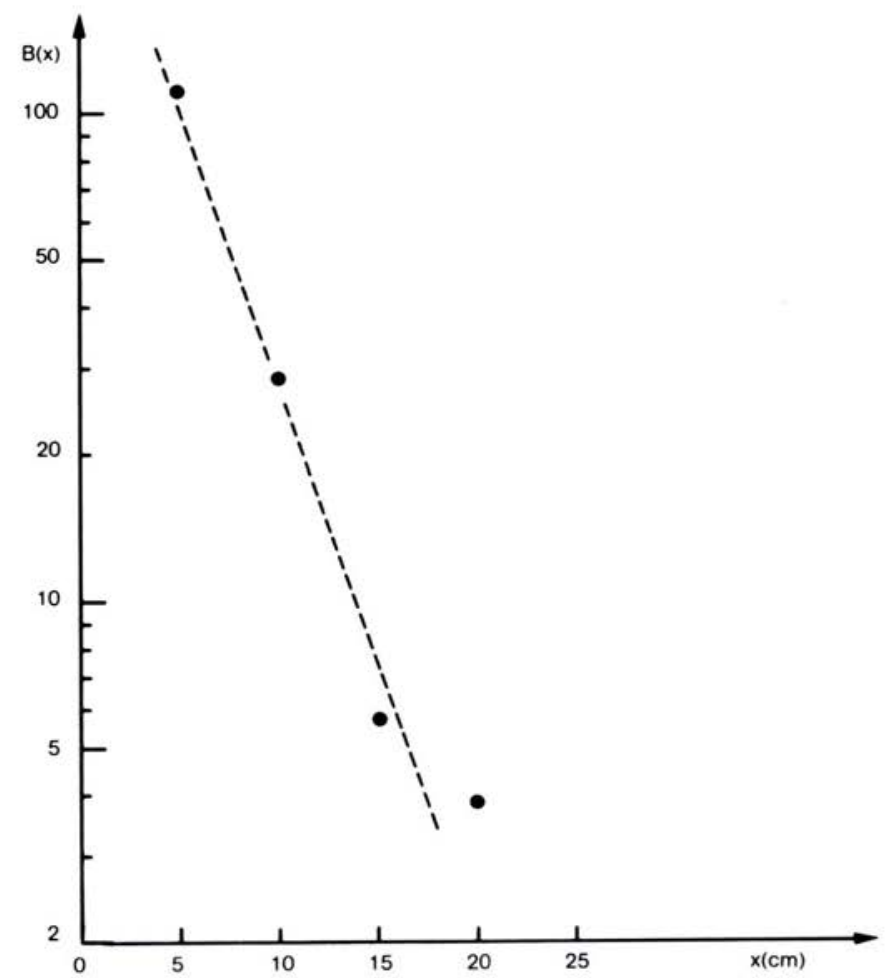

Fig. 3. - Distribution en fonction de la profondeur de sol non perturbé de $B(x)$ (équation 2).

Lieu: ferme expérimentale de l'université de Thessalonique.

II est intéressant de comparer nos résultats à la radioactivité naturelle de ${ }^{40} \mathrm{~K}$ dans le sol. Pour tous les départements de la Grèce du nord, la radioactivité de ${ }^{134} \mathrm{Cs}$ et ${ }^{137} \mathrm{Cs}$ reste presque toujours inférieure à celle de ${ }^{40} \mathrm{~K}$, même entre 0 et $10 \mathrm{~cm}$. La concentration de ${ }^{40} \mathrm{~K}$ dépendant très peu de la profondeur, nous voyons que, pour des profondeurs supérieures à $10 \mathrm{~cm}$, l'activité de ${ }^{134} \mathrm{Cs}$ et ${ }^{137} \mathrm{Cs}$ devient négligeable par rapport à celle de ${ }^{40} \mathrm{~K}$.

\section{CONCLUSION}

Nous avons déterminé de manière indirecte le dépôt de radiocésium sur la Grèce du nord, après Tchernobyl. La concentration de ${ }^{137} \mathrm{Cs}$ diminue exponentiellement en fonction de la profondeur; $95 \%$ du radiocésium déposé est toujours piégé dans les 10 premiers centimètres d'un sol non perturbé. L'activité naturelle de ${ }^{40} \mathrm{~K}$ dans le sol est toujours supérieure à celle du radiocésium déposé, même entre 0 et $10 \mathrm{~cm}$ de sol non perturbé. 


\section{RÉFÉRENCES}

[1] COMMISSARIAT À L'ÉNERGIE ATOMIQUE HELLENIQUE. L'accident nucléaire de Tchernobyl et ses conséquences en Grèce. Rapport $n^{\circ}$ 1. Athènes, juillet 1986.

[2] COMMISSARIAT Ȧ L'ÉNERGIE ATOMIQUE HELLENIQUE. L'accident nucléaire de Tchernobyl et ses conséquences en Grèce. Rapport $n^{\circ} 2$. Athènes, décembre 1986.

[3] ROGOWSKI A., TAMURA T. Erosional behaviour of cesium 137. Health Phys., 1970, 18, 467-477.

[4] UNITED NATIONS SCIENTIFIC COMMITTEE ON THE EFFECTS OF IONIZING RADIATION. Sources and biological effects, report to the general assembly (ONU Publications, document $\mathrm{n}^{\circ}$ E82 1x8). New York: United Nations, 1982. 